International Journal of Pure and Applied Mathematics

Volume 95 No. 2 2014, 131-135

ISSN: 1311-8080 (printed version); ISSN: 1314-3395 (on-line version)

url: http://www.ijpam.eu

doi: http://dx.doi.org/10.12732/ijpam.v95i2.2

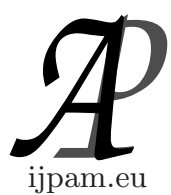

\title{
A GROUP WHERE C-PERMUTABILITY DOES NOT IMPLY C-NORMALITY
}

\author{
Doaa Mustafa Alsharo ${ }^{1}$, Hajar Sulaiman ${ }^{2}$ \\ ${ }^{1}$ School of Mathematical Sciences \\ Universiti Sains Malaysia \\ 11800, USM, Penang, MALAYSIA \\ ${ }^{2}$ School of Mathematical Sciences \\ Universiti Sains Malaysia \\ 11800, USM, Penang, MALAYSIA
}

\begin{abstract}
The $c$-permutability of subgroups in a finite group was recently discovered and a number of related results have been published. As there is a close relationship between permutability and normality, it is not surprising that a property called $c$-normality was defined and linked with $c$-permutability. By definition, all $c$-normal subgroups are $c$-permutable. In this paper, we show that the converse is not true by creating a group of order 32 containing a $c$-permutable subgroup that is not $c$-normal.
\end{abstract}

AMS Subject Classification: 20D10

Key Words: permutable subgroups, c-normal subgroups, c-permutable subgroups

\section{Introduction}

We first note that all groups and subgroups considered in this paper are finite.

Received: February 12, 2014

(C) 2014 Academic Publications, Ltd.

$\S$ Correspondence author url: www.acadpubl.eu 
A subgroup $H$ of a group $G$ permutes with a subgroup $K$ of $G$ if $H K$ is also a subgroup of $G$. In addition, if $H$ permutes with every subgroup of $G$ then $H$ is said to be a permutable subgroup of $G$. By [3], every permutable subgroup $H$ of $G$ is subnormal. The study of permutable subgroups has resulted to many interesting properties, especially in the finite cases.

$c$-normality and $c$-permutability are two properties that was discovered and defined by Wang [5] and Al-sharo [2], respectively. Wang uses $c$-normality, which is a "weaker" version of normality, to generalize some known theorems. In particular, he proved that a group $G$ is solvable if and only if every maximal subgroup of $G$ is $c$-normal in $G$. Al-sharo instead proved that a group $G$ is solvable if and only if every maximal subgroup of $G$ is $c$-permutable in $G$. Studies have also been done on transitivity of these properties. In [2], Al-sharo introduced the CPT-group, a group in which $c$-permutability is a transitive relation. Transitive relation here means that for all subgroups $H$ and $K$ of $G$, if $H$ is $c$-permutable in $K$ and $K$ is $c$-permutable in $G$, then it implies $H$ is $c$-permutable in $G$. Alsharo [1] have instead studied on $C T$-groups, i.e groups in which $c$-normality is a transitive relation. They stated and proved a number of new characterizations of finite solvable $C T$-groups.

From [2] and [5], we deduce that if $G$ is a solvable group then every maximal subgroup of a group $G$ is $c$-normal and $c$-permutable. From the definitions of $c$-normal and $c$-permutable subgroups, it is known that every $c$-normal subgroup of a group $G$ is $c$-permutable. In this paper, we show that the converse is not true. The Groups, Algorithms and Programming (GAP) software was used for the computations.

\section{Preliminaries}

We assume that the reader has prior knowledge of normality and subnormality of subgroups. Here, we give some preliminaries on $c$-normality and $c$-permutability of subgroups in a finite group $G$.

Definition 2.1. (see [5]) Let $H$ be a subgroup of a finite group $G$. The core of $H$ in $G$, denoted as $H_{G}$, is defined to be the largest normal subgroup of $G$ contained in $H$, (or equivalently to $H_{G}=\cap\left\{H^{g}: g \in G\right\}$ ).

Definition 2.2. (see [5]) Let $G$ be a group. We will call a subgroup $H$ of $G c$-normal in $G$ if there exists a normal subgroup $N$ of $G$ such that $H N=G$ and $H \cap N \leq H_{G}$. $H c$-norm $G$ denotes $H$ is normal in $G$. 
Wang [5] deduced that every normal subgroup of a finite group $G$ is $c$-normal. However, the following example shows that the converse is not true.

Example 2.3. Let $G=S_{3}$. Consider $H=<(12)>$, a Sylow 2-subgroup of $G$. Now, $H$ is $c$-normal in $G$ since $H A_{3}=S_{3}$ and $H \cap A_{3}=1 \leq H S_{3}=1$. However, since $H$ is not subnormal in $G$, then $H$ is not a normal subgroup of $G$.

Definition 2.4. (see [2]) Let $H$ be a subgroup of a finite group $G$. The ${ }_{p}$ Core of $H$ in $G$ is defined to be the largest permutable subgroup of $G$ contained in $H$, and denoted by ${ }_{p} \operatorname{Core}_{G}(H)$, or $H_{P G}$.

Definition 2.5. (see [2]) Let $G$ be a group. We call a subgroup $H$ $c$-permutable if there exists a permutable subgroup $P$ of $G$ such that $H P=G$ and $H \cap P \leq H_{P G}$. We use $H c$-perm $G$ to denote $H$ is $c$-permutable in $G$.

Permutable subgroups are $c$-permutable but the converse is not true. The following example shows this.

Example 2.6 The symmetric group $S_{3}$, take $H$ the Sylow 2-subgroup of $S_{3}$, we can see that $H$ is not permutable, but it is $c$-permutable with all subgroups of $S_{3}$.

It has been proven that a normal subgroup is permutable. Therefore, since a normal subgroup is always $c$-normal, then a $c$-normal subgroup must be permutable and hence is $c$-permutable. What follows is a computational proof, using $G A P$, showing that a $c$-permutable subgroup need not be $c$-normal.

\section{Main Result}

To prove that $c$-permutability does not imply $c$-normality, we create a group $G$ of order 32 and list its subgroups. We show that all subgroups of $G$ are $c$-permutable but there exists among them a subgroup which fails to be $c$-normal.

Theorem 3.7. Let $G$ be a group with all its subgroups $c$-permutable. Then, there exists a subgroup $H$ of $G$ where $H$ is not $c$-normal.

Proof. Let $G=<a, b, c, a^{8}=b^{2}=c^{2}=1, b^{a}=b c, c^{a}=c a^{4},\left(a^{2}\right)^{b}=b a^{4}>$ 
The order of $G$ is $32=2^{5}$, and $G$ is isomorphic to the semidirect product: $\left(Z_{8} \rtimes Z_{2}\right) \rtimes Z_{2}$

Now since $p$-groups are nilpotent and every subgroup of a nilpotent group is subnormal then every subgroup of $G$ is subnormal.

We use Groups, Algorithms Programming (GAP) for the computation, we see that $G$ has 42 subgroups. They are:

$$
\begin{aligned}
& <>,<a^{4}>,<a^{7} b a b>,<a^{6} b a b a>,<b>,<a^{-1} b a>,<a^{-2} b a^{2}>, \\
& <a^{-3} b a^{3}>,<a^{6} b>,<a^{5} b a>,<b^{-1} a^{6} b^{2}>,<b^{-1} a^{5} b a b><a^{-2}>, \\
& <c, a c a^{-1}>,<a^{2} c^{-1}>,<b, a^{-4}>,<a^{-1} b a, a^{-4}>,<a^{2} b^{-1}, a^{-2} b^{-1}>, \\
& <a b^{-1} a, a^{-3} b^{-1} a>,<c, a^{-2} b^{-1}>,<b^{-1} c b, b^{-1} a^{-2}>, \\
& <b^{-1} a^{-1} c a b, b^{-1} a^{-3} b^{-1} a b>,<a^{-1} c a, a^{-3} b^{-1} a>,<b, c>,<a^{-3} b a^{3}, a^{-3} c a^{3}> \\
& ,<a^{-2} b a^{2}, a^{-2} c a^{2}>,<a^{-1} b a, a^{-1} c a>,<b, c, a b a^{-1}>,<a^{-2}, c>, \\
& <c, a^{2} b^{-1} a c a^{-1}>,<a^{-2}, b>,<a^{-2}, a^{-1} b a>,<b, a^{2} c^{-1}>, \\
& <a^{-1} b a, a c^{-1} a>,<a>,<b^{-1} a b>,<b a^{-1}>,<a^{-1} b>,<a^{-2}, b, c>, \\
& <a, c>,<a^{-2}, b a^{-1}>,<G>.
\end{aligned}
$$

All these subgroups are $c$-permutable in $G$. But if we take $H=<a^{7} b a b>=$ $\left\{1, a^{7} b a b\right\} \simeq Z_{2}$ subgroup of order 2 , then $H$ is not normal in $G$. Since $a H \neq H a$, and $H$ is contained in every subgroup of order 6 , then $H$ cannot be $c$-normal in $G$.

Hence, there is a $c$-permutable subgroup $H$ of $G$ that is not $c$-normal.

\section{Conclusion}

We have shown that there exists a group $G$ of order 32 for which $c$ - permutability does not imply $c$-normality. Hence, this proves that $c$-permutability does not imply $c-$ normality.

\section{References}

[1] D.M. Alsharo, H. Sulaiman, K.A. Al-Sharo, I. Suleiman, A note on finite groups in which $C$-Normality is a transitive relation, International Mathematical Forum, 8 (2013), 1881-1887, doi: 10.12988/imf.2013.39168.

[2] K.A. Al-Sharo, I. Suleiman, A note on finite groups in which c-permutability is transitive, Acta Mathematica Hungarica, 134 (2012), 162-168, doi: 10.1007/s10474-011-0132-0.

[3] O.H. Kegel, Sylow-Gruppen und Subnormaleiler endlicher Gruppen, Math. Z., 78 (1962), 205-221, doi: 10.1007/BF01195169. 
[4] O. Ore, Contributions in the theory of groups of finite order, Duke Math. J., 5 (1939), 431-460, doi: 10.1215/s0012-7094-39-00537-5.

[5] Y. Wang, C-normality of groups and its properties, Journal of Algebra, 180 (1996), 954-965, doi: 10.1006/jabar.1996.0103. 
